\title{
BMJ Open Active living environments, physical activity and premature cardiometabolic mortality in Canada: a nationwide cohort study
}

\author{
Sarah M Mah (D) , ${ }^{1}$ Claudia Sanmartin, ${ }^{2}$ Mylène Riva, ${ }^{1,3}$ Kaberi Dasgupta, ${ }^{4,5}$ \\ Nancy A Ross ${ }^{1}$
}

To cite: Mah SM, Sanmartin C, Riva M, et al. Active living environments, physical activity and premature cardiometabolic mortality in Canada: a nationwide cohort study. BMJ Open 2020;10:e035942. doi:10.1136/ bmjopen-2019-035942

- Prepublication history and additional material for this paper are available online. To view these files, please visit the journal online (http://dx.doi. org/10.1136/bmjopen-2019035942).

Received 25 November 2019 Revised 03 September 2020 Accepted 30 September 2020

\section{Check for updates}

(c) Author(s) (or their employer(s)) 2020. Re-use permitted under CC BY-NC. No commercial re-use. See rights and permissions. Published by BMJ.

1Department of Geography, McGill University, Montreal, Quebec, Canada

${ }^{2}$ Statistics Canada, Ottawa, Ontario, Canada

${ }^{3}$ Institute for Health and Social Policy, McGill University, Montreal, Quebec, Canada ${ }^{4}$ Department of Medicine, McGill University, Montreal, Quebec, Canada

${ }^{5}$ Centre for Outcomes Research and Evaluation, Research Institute of the McGill University Health Centre, Montreal, Quebec, Canada

Correspondence to Dr Nancy A Ross; nancy.ross@mcgill.ca

\section{ABSTRACT}

Objective To evaluate sex-specific and age-specific associations of active living environments (ALEs) with premature cardiometabolic mortality.

Design Population-based retrospective cohort study. Setting Residential neighbourhoods (1000-metre circular buffers from the centroids of dissemination areas) across Canada for which the Canadian ALE Measure was derived, based on intersection density, points of interest and dwelling density.

Participants 249420 survey respondents from an individual-level record linkage between the Canadian Community Health Survey (2000-2010) and the Canadian Mortality Database until 2011, comprised of older women (65-85 years), older men (65-81 years), middle-aged women (45-64 years) and middle-aged men (45-64 years).

Primary outcome measures Premature cardiometabolic mortality and average daily energy expenditure attributable to walking. Multivariable proportional hazards regression models were adjusted for age, educational attainment, dissemination area-level median income, smoking status, obesity, the presence of chronic conditions, season of survey response and survey cycle.

Results Survey respondents contributed a total of 1 451913 person-years. Greater walking was observed in more favourable ALEs. Walking was associated with lower cardiometabolic death in all groups except for middle-aged men. Favourable ALEs conferred a $22 \%$ reduction in death from cardiometabolic causes (HR 0.78, 95\% Cl 0.63 to 0.97) for older women.

Conclusions On average, people walk more in favourable ALEs, regardless of sex and age. With the exception of middle-aged men, walking is associated with lower premature cardiometabolic death. Older women living in neighbourhoods that favour active living live longer.

\section{INTRODUCTION}

There is growing interest in the role that environments might play in supporting active living and reducing the health burden associated with sedentary lifestyles. Physical inactivity is a large contributor to reduced life expectancy in Canada. ${ }^{1}$ A high proportion of cardiometabolic death could be avoided
Strengths and limitations of this study

- This is the first study to demonstrate individuallevel associations between favourable active living environments, greater walking and lower premature cardiometabolic death in Canada.

- Strengths include a large national sample, long follow-up time, objectively derived measures for the active living environment, and the inclusion of individual-level characteristics and health behaviours.

- Due to the cross-sectional nature of the survey, residential moves could not be accounted for. Whether respondents' reported walking was performed in their neighbourhoods of residence is unknown.

- Retrospective data are unable to address the reasons why an association is only present for older women and signal a need for further research.

if those who were physically inactive became physically active. ${ }^{2}$ Neighbourhoods that support active living could help reduce sedentary lifestyles, but have not been conclusively linked to reductions in premature cardiometabolic mortality.

Active living environments (ALEs), also widely known as 'walkable environments', are places that are easily navigable with wellconnected walking paths, have a number and variety of destinations, and are more densely populated. ${ }^{3}$ These neighbourhoods are understood to encourage active livingthe kind of neighbourhood-based activity that tends to be 'built-in' to one's daily life. Studies have established links between ALEs and obesity ${ }^{4}$ as well as cardiometabolic risk factors such as hypertension ${ }^{5}$ and type 2 diabetes. ${ }^{6}$ Higher walking levels are associated with lower mortality in older adults, ${ }^{8}$ and these benefits are likely driven by reductions in cardiometabolic mortality. 
The ALE's impact on cardiometabolic disease is likely subject to life-course changes in physical activity and cardiometabolic risk from midlife to old age. ${ }^{9}$ The mechanism and extent to which these environments encourage active living and reduce premature cardiometabolic mortality might also differ between the sexes due to biological variations in cardiometabolic risk, ${ }^{10}$ differences in premature mortality-given overall life expectancy for women is higher than for men, as well as gendered social factors that influence health behaviours.

Few studies have assessed relationships between neighbourhood characteristics and cardiometabolic mortality. ${ }^{11-13}$ Those that have tend to be ecological studies, and have reported inconclusive or conflicting associations between the built environment and mortality. ${ }^{14-16}$ In this study, we determined whether favourable ALEs are associated with lower risk of premature cardiometabolic mortality through the physical activity pathway in groups that are stratified by sex and age.

\section{METHODS}

\section{Study population}

We used an individual-level record linkage between the Canadian Community Health Survey (CCHS) cycles 20002011 and the Canadian Mortality Database (CMDB) years 2000-2011. The linked dataset contains 614755 respondents with follow-up time of up to 11.32 years from survey response to 31 December 2011 or death. The CCHS is an annual, national cross-sectional survey of approximately 65000 people aged 12 years and older sampled from the community-dwelling Canadian population. Response rates for the CCHS exceeded $70 \%$ for these cycles. ${ }^{17}$ The CCHS is linked to CMDB, ${ }^{18}$ the registry for all deaths recorded in Canada which contains date of death and primary cause of death, coded by the International Classification of Disease (ICD). ${ }^{19}$

Analyses were stratified by sex and age (at baseline) into older women aged $65-85$ years ( 85 years being the average life expectancy for Canadian women), ${ }^{20}$ older men aged 65-81 years (81 years being the average life expectancy for Canadian men), ${ }^{20}$ middle-aged women $45-64$ years and middle-aged men 45-64 years. Low cardiometabolic death rates were seen for younger adults and were not examined. In order to set a minimum period of exposure to neighbourhoods in Canada, we excluded recent immigrants (in the past 5 years), as well as those with a follow-up time of less than 1 year between survey response and censoring or death.

\section{Active living environments}

The Canadian ALEs Database ${ }^{21}$ (Can-ALE) is comprised of geographical measures for Canadian neighbourhoods derived from open data. Briefly, the measures are constructed using 1000-metre circular (Euclidean) buffers from the centroids of dissemination areas (DAs), the smallest geographical area for which complete census data are released. DAs correspond to roughly 400-700 persons. Three components are included in the measure: intersection density, points of interest and dwelling density. Intersection density (the number $\geq 3$-way intersections) was derived using OpenStreetMap 2016 road and footpath features. The number of points of interest was also retrieved from OpenStreetMap 2016. Dwelling density was obtained from the 2016 Canadian census. Raw scores for the three components were then clustered using k-medians and grouped into five categories representing environments that are very active living-unfriendly (class 1 ) to those that are very active living-friendly (class 5). We also derived a two-component 2006 score (which lacks points of interest from 2006) that was closer to baseline survey response and confirmed that little change occurred between 2006 and 2016 scores for the ALE. We interpolated residential locations based on reported postal code in the CCHS. Statistics Canada's Postal Code Conversion File Plus is a program that assigns spatial coordinates based on population-weighted random allocation. We overlaid these coordinates with 2016 DAs in a Geographic Information System (GIS, ArcGIS V.10, ESRI 2010), and assigned areas their corresponding Can-ALE classification.

\section{Walking measures}

We approximated average daily energy expenditure related to leisure-time walking and to leisure-time physical activity for each respondent, which we refer to as 'walking' and 'physical activity', respectively. Daily energy expenditure for leisure-time physical activity is available as a derived variable in the CCHS and has been described previously. ${ }^{22}$ Average daily energy expenditure for walking was derived using the same method. Briefly, the calculation is based on respondents' self-reported activities in the last 3 months from baseline survey response. Frequency and time spent walking were multiplied by an assigned metabolic equivalents value of 3 for walking. We then divided this number by 365 days to yield daily energy expenditure in $\mathrm{kcal} / \mathrm{kg} /$ day. We hypothesised that favourable ALEs specifically enable walking as a neighbourhood-based physical activity.

\section{Outcomes}

The cause of interest was death due to metabolic diseases (ICD 10 codes E11-E14, E65-68, E78) or cardiovascular conditions (I10-15, I21-25, I50, I61-69, I70-74) which we term cardiometabolic mortality. Premature death has been defined as death before reaching 75 years, ${ }^{23}$ and since the maximum age in the middle-aged groups at baseline was 64 years, the maximum age reached for middle-aged men and women was 72 years at censoring. To assess deaths that were premature in nature for older age groups, we analysed deaths that occurred before average life expectancy for women and for men in Canada by censoring at 85 years and 81 years, respectively. ${ }^{20}$ We modelled these four groups separately to allow us to observe differences in physical activity levels, cardiometabolic risk trajectories and potential response to the built environment. 


\section{Covariates}

Covariates with known associations with mortality were selected a priori. These included age, educational attainment, DA-level median income, smoking status, obesity, and the presence of two or more chronic conditions. We also adjusted for survey cycle and season of survey response for models that included average daily energy expenditure related to walking or physical activity. Body mass index was adjusted for systematically overestimated height and underestimated weight using previously established correction factors, ${ }^{24}$ which we then used to classify 'obesity' as $30 \mathrm{~kg} / \mathrm{m}^{2}$ and above. The presence of two or more chronic conditions was ascertained based on CCHS responses to items in the chronic diseases module that were consistent across survey cycles.

\section{Statistical analysis}

First, we examined whether respondents who lived in ALEs reported higher walking or overall physical activity levels using descriptive statistics (online supplemental figure S1). Trends across the five ALE classes were evaluated by entering the categorical ALE variable as an ordinal variable in a generalised linear model with a log link, adjusted for individual-level factors. Second, we assessed whether walking is associated with premature cardiometabolic death for women and men stratified across middle and older age groups using Cox proportional hazards models. Due to the skewed non-negative nature of energy expenditure, we entered daily energy expenditure for walking into the model as a three-level factor variable, consisting of: no walking (reference), less than $1.44 \mathrm{kcal} /$ $\mathrm{kg} /$ day and more than or equal to $1.44 \mathrm{kcal} / \mathrm{kg} /$ day. This cut-off is equivalent to the recommended $150 \mathrm{~min}$ or more per week of moderate to vigorous exercise, ${ }^{25}$ which we applied to walking alone, and to overall physical activity (online supplemental figure S2) for sample calculations. Finally, we assessed whether associations between favourable ALEs and lower premature cardiometabolic mortality could be detected using Cox proportional hazard models.

All models were adjusted for age, education, area-level income, survey cycle, smoking status, obesity, and the presence of two or more chronic conditions. Cox models assessing associations between walking and premature cardiometabolic mortality were also adjusted for season of survey response. Due to low sample sizes and events in ALE class 5, ALE classes 1-3 were aggregated as less favourable, while classes 4 and 5 were aggregated as more favourable for survival analyses. The proportional hazards assumption was assessed for ALEs using visual inspection of Schoenfeld residuals as well as assessing for similarity between Cox predicted curves and observed KaplanMeier curves. Age was entered as a continuous linear variable after testing quadratic and cubic terms, evaluating Martingale residuals, as well as examining interactions with time for potential time-varying behaviour-none of which impacted the final estimates for the ALE in any group. Competing risks models were also used to assess the impact of non-cardiometabolic premature death on estimates for favourable ALEs. Note that in accordance with confidentiality protocols, counts are reported using a rounding base of five, and therefore may not be additive to rounded totals.

\section{Patient and public involvement}

Participants and members of the public were not involved in the design, analysis or interpretation of this study. However, the research question is of broad policy relevance and public health interest. The results will be disseminated to the general public, policymakers and stakeholders through websites, seminars and conferences.

\section{RESULTS}

Of the 614755 linked CCHS respondents (figure 1), 311 090 who were under 45 years old or recently immigrated to Canada were removed from the analysis. Of the 303 685 remaining, 268210 respondents $(87.9 \%)$ had at least 1 year of follow-up and were considered eligible for inclusion in the study. Of those eligible, 249420 respondents (93.0\%) had complete information (missing data are summarised in online supplemental table S1).

Among 249420 individuals with a grand total of 1451 913 person-years, the total follow-up time by sex and age group ranged from 177480.84 person years in older men (median follow-up time 4.47 years) to 522885.69 person years in middle-aged women (median follow-up time 6.39 years) (table 1). The proportion of those who died from cardiometabolic causes before age 72 was over twofold higher for middle-aged men (830 deaths, $1.1 \%$ ) than for middle-aged women (385 deaths, $0.5 \%$ ). Baseline characteristics of the entire study population stratified by ALE revealed that the most favourable environments tended to have the lowest proportion of obese respondents, the highest proportion of highly educated respondents and the lowest area-level household income on average (online supplemental table S2).

\section{Gradation of walking with ALEs}

Mean daily energy expenditure for walking was highly graded by the ALE in all groups ( $\mathrm{P}$ for trend $<0.01$ for all groups, figure 2), driven in part by the high proportions of inactive respondents (ie, those who report never walking) living in the least favourable Can-ALE classes. In contrast, overall physical activity was not graded by the favourability of the ALE (online supplementary figure S3), except for an inverse graded pattern in middle-aged women $(\mathrm{P}<0.001)$. Older women and older men exhibited the largest gains in average daily energy expenditure for walking between the least favourable and most favourable neighbourhoods at $56 \%(0.63-0.98 \mathrm{kcal} / \mathrm{kg} /$ day $)$ for older women and $64 \%(0.72-1.18 \mathrm{kcal} / \mathrm{kg} /$ day $)$ for older men. The proportion of overall physical activity attributable to walking was high for older women in particular (figure 2). 


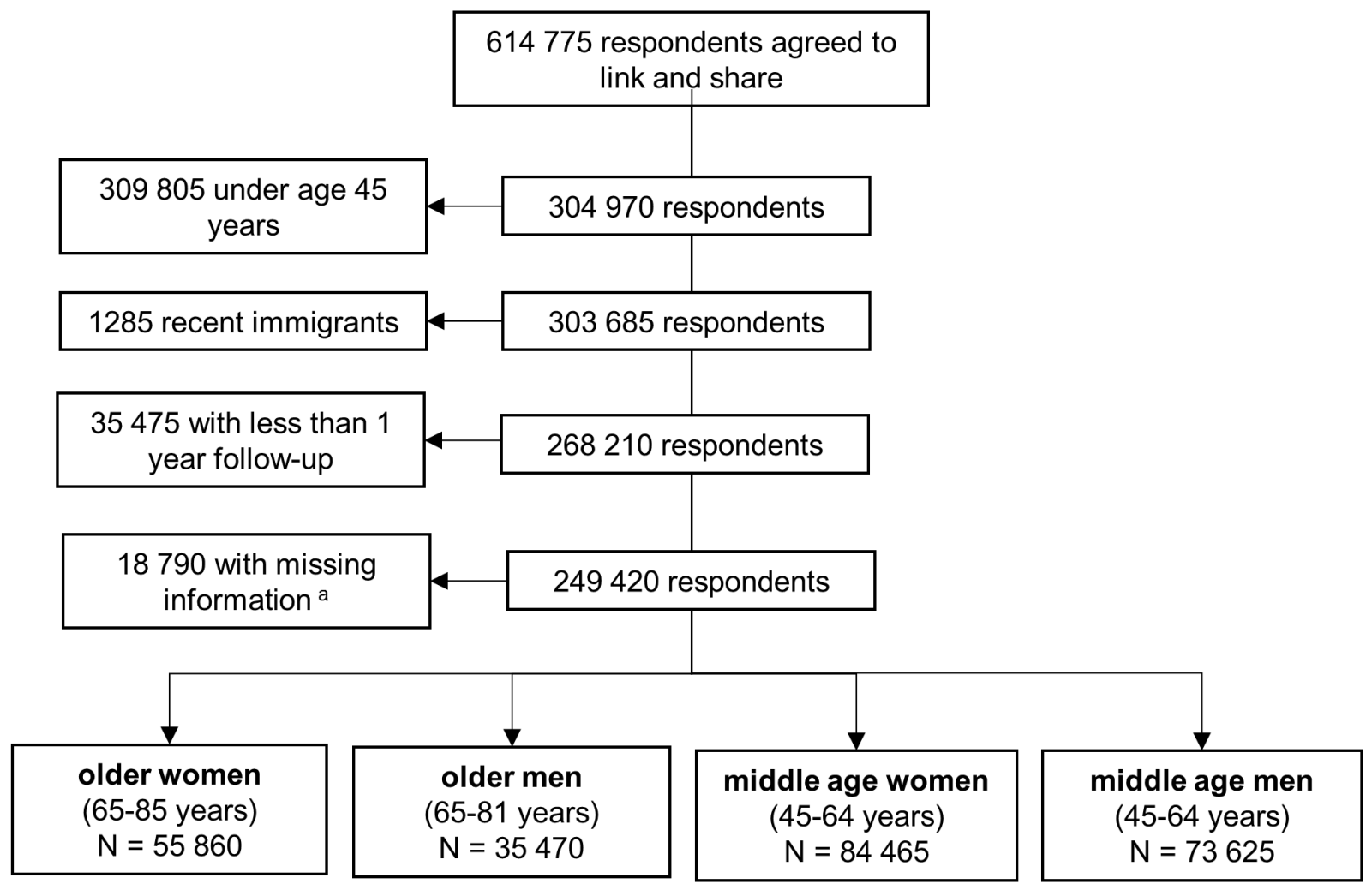

Figure 1 Flowchart of participant inclusion. ${ }^{a}$ Missing data are summarised in online supplemental table S1.

\section{Association of walking with premature cardiometabolic mortality}

Higher and lower levels of walking compared with no walking were associated with lower risk of premature cardiometabolic death for all groups except middle-aged men (comparing the highest level of walking with no walking-older women: HR $0.68,95 \%$ CI 0.57 to 0.80 ; older men: HR $0.65,95 \%$ CI 0.55 to 0.77 ; middle-aged women: HR $0.57,95 \%$ CI 0.42 to 0.78 ; middle-aged men: HR $1.02,95 \%$ CI 0.83 to 1.24 ), and was most apparent in middle-aged women (figure 3). Associations between overall physical activity and premature cardiometabolic mortality were found for all groups (online supplemental figure S4).

Association of ALEs with premature cardiometabolic mortality We identified an inverse association between more favourable environments and premature cardiometabolic mortality for older women, after adjusting for individuallevel factors (HR $0.78,95 \%$ CI 0.63 to 0.97 , figure 4 ). No conclusive associations were observed for older men (HR $1.13,95 \%$ CI 0.90 to 1.41), middle-aged women (HR 1.02, 95\% CI 0.68 to 1.54 ) or middle-aged men (HR 0.97, 95\% CI 0.73 to 1.30 ). The proportional hazards assumption held, based on visual inspection of Schoenfeld residuals and similarities between Cox predicted curves and Kaplan-Meier curves. Competing risks models yielded similar estimates to Cox proportional hazards models (online supplemental table S3).

\section{DISCUSSION}

To our knowledge, this is the first individual-level study to examine associations between ALEs and premature cardiometabolic death. We demonstrated that older women living in favourable ALEs were 22\% less likely to die prematurely from cardiometabolic diseases than those living in less favourable environments. Older women living in these neighbourhoods walked more, and their walking-related energy expenditure was inversely associated with premature cardiometabolic mortality. The fact that these associations were robust to adjustment for social class (which, for measures such as income, were inversely related to ALE favourability, online supplemental table S2) speaks to the importance of neighbourhoods in shaping behaviours and health, over and above the sociodemographic composition of the people living in the neighbourhood. In other groups, the association between the ALE and premature cardiometabolic mortality did not hold. There were, however, positive associations between the ALE and walking, regardless of sex and age. We also observed inverse associations between walking and premature cardiometabolic mortality in 
Table 1 Characteristics of study population $(n=249420)$

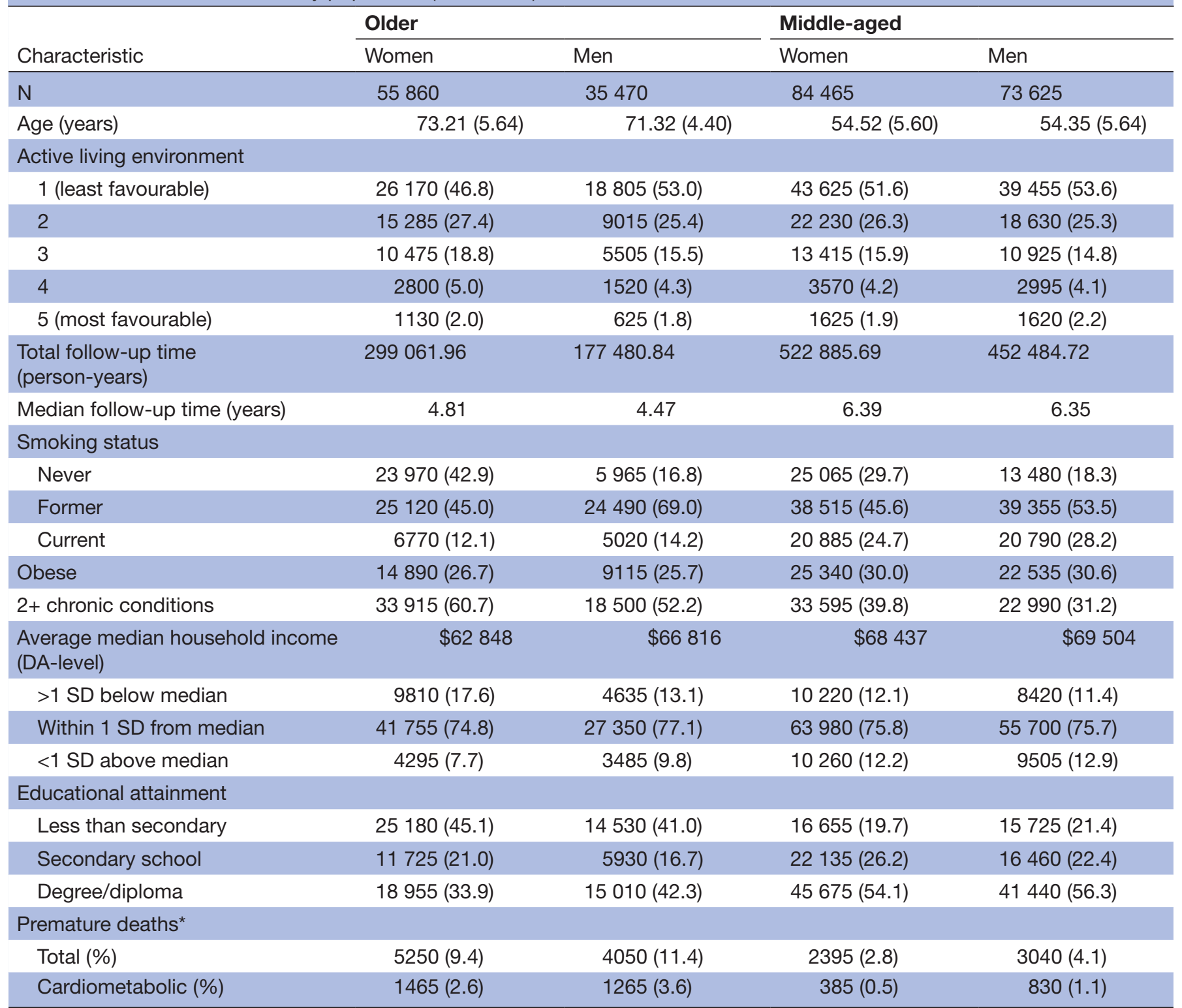

Data are $\mathrm{n}, \mathrm{n}(\%)$, mean (SD). All covariates are from survey response (baseline).

${ }^{*}$ Premature death was defined as death before the age of 85 years in older women, before 81 years in older men, and before 72 years in middle-aged women and men.

DA, dissemination area.

all groups except for middle-aged men (online supplemental figure S5).

Consistent with previous studies in Canada, ${ }^{26} 27$ we found incrementally higher walking levels in more favourable ALEs. The larger increases in walking for older adults living in favourable neighbourhoods relative to middle-aged adults might point to the growing importance of walking with age. ${ }^{28}$ Older adults often have a smaller, more restricted activity space than younger and middle-aged adults, and may spend more time where they live. ${ }^{29}$ There is also consistent evidence that more women walk for leisure than men, and might also walk more for the purpose of running errands. ${ }^{30}$ Although older men report higher daily energy expenditures for walking, older women report the highest and most environmentally graded proportions of physical activity attributable to walking. Some women of previous generations never drove, and while the gap between men and women holding a driver's licence has since narrowed, older men are still more likely to drive as a main mode of transportation. ${ }^{31}$ This could explain some of the discrepancies we see between women and men. Nevertheless, the strong relationship between walkable environments and walking for women has been shown using large-scale data, ${ }^{32}$ and signals the important role that the ALE could play for older women to achieve adequate levels of physical activity. 

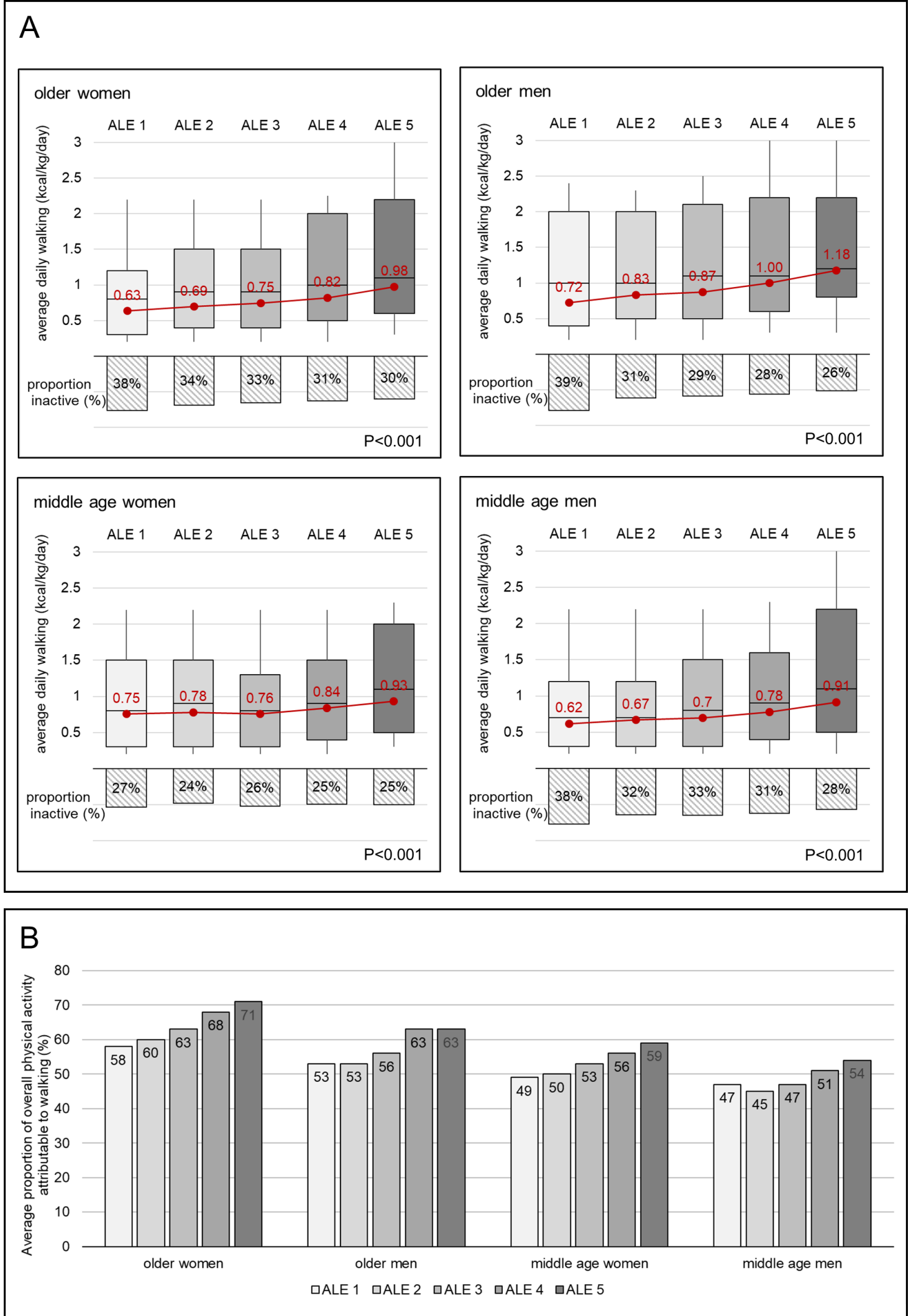

Figure 2 Average daily energy expenditure related to walking. Data are mean or (\%). Active living environment (ALE) 1 represents the least favourable environment, while ALE 5 represents the most favourable environment. (A) Contains boxplots, mean energy expenditure related to walking and proportion that report no walking. Boxes represent the IQR (25th-75th percentile) and the horizontal line represents the median. Note that upper and lower limits of the boxplots have been adjusted to represent the 90th and 10th percentile, respectively, for confidentiality purposes. Red markers and trend line represent means in each ALE class with test for trend $(P<0.05)$. (B) Shows the proportion of all physical activity that walking accounts for, by ALE favourability. 


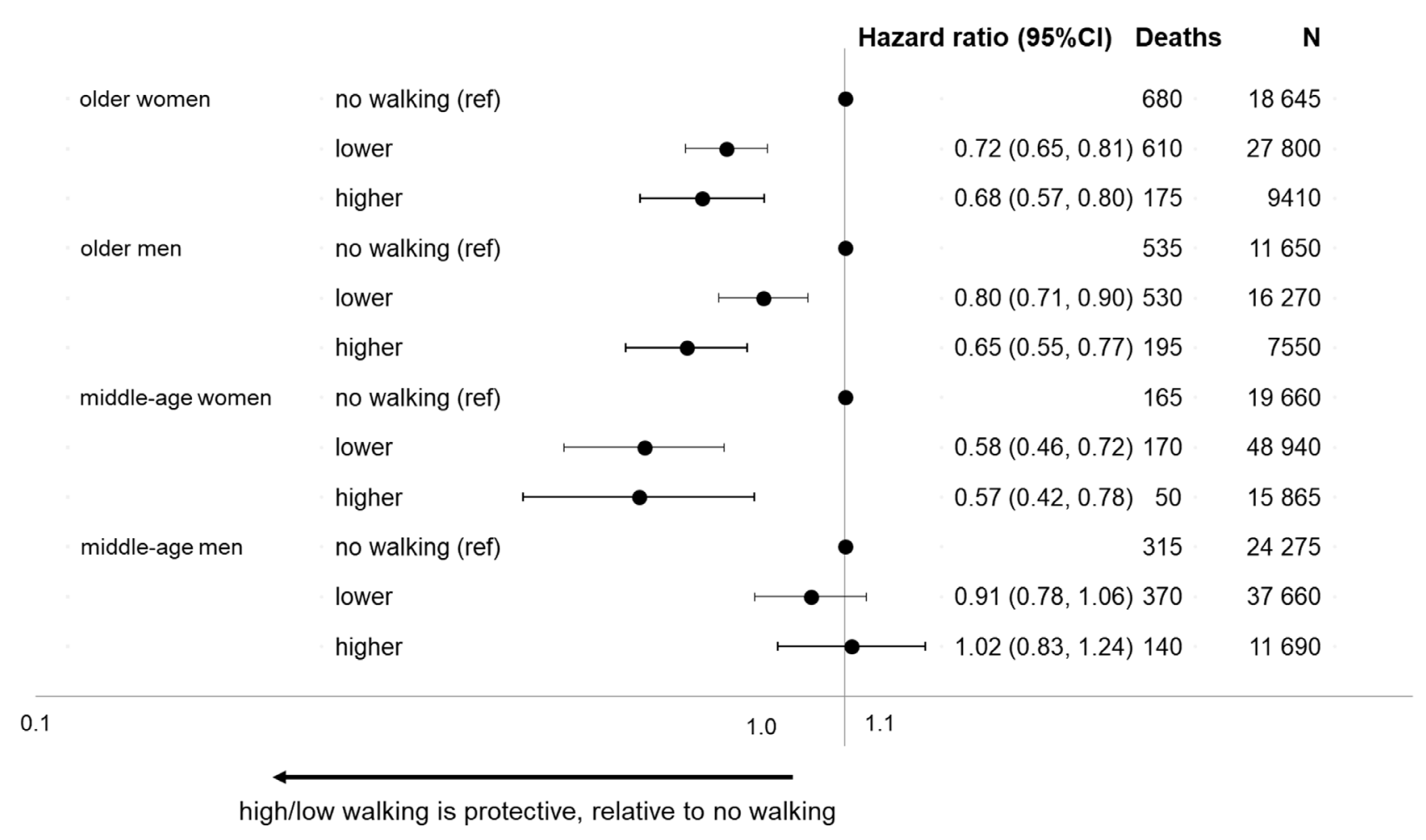

Figure 3 Association of walking with premature cardiometabolic mortality. Data are HRs $(95 \% \mathrm{Cl})$. Models are adjusted for baseline age, education, income, the presence of two or more chronic conditions, obesity, season of survey response and survey cycle.

The associations between leisure walking and survival benefit that we observed in this cohort complement previous work demonstrating the importance of overall leisure-time physical activity to mortality. ${ }^{33}$ The notable absence of association between walking and favourable cardiometabolic outcomes in middle-aged men could be a result of greater participation in sports and other activities ${ }^{30}$ given the protective association between overall physical activity and premature cardiometabolic mortality we observed for this group. Walking had the highest inverse association (43\% risk reduction of premature cardiometabolic death) in middle-aged women. A recent systematic review of 36 studies found that more women walk for leisure than do men-particularly in younger age groups. ${ }^{34}$ It is possible that the exercise achieved through activities built into daily living endures over the life course and could place women on an advantageous trajectory when it comes to long-term cardiometabolic health outcomes.

Our findings point to a potential avenue for preventing premature cardiometabolic mortality in older womenthe importance of which is underlined by the stalls in improving cardiovascular outcomes and mortality for women compared with the improvements observed for men. ${ }^{35}$ These neighbourhoods might support activities of daily living that will later translate into survival benefits for older women. ${ }^{36}$ The inverse association between the
ALE and premature cardiometabolic death corroborates findings of a previous study which demonstrated a similar relationship between urban compactness and coronary heart disease death in a biomedical cohort of postmenopausal women. ${ }^{13}$ Our study is also consistent with previous research that links favourable environments to modest reductions in area-level rates of premature cardiovascular disease mortality, ${ }^{14}$ ischaemic heart disease ${ }^{12}$ and overall mortality. ${ }^{11}{ }^{16}$ Past research has been limited to ecological studies, area-level socioeconomic variables and small sample sizes. Our national sample, paired with GIS-derived measures for ALEs, offers the advantage of comprehensive individual-level information to assess the built environment's potential for influencing downstream health outcomes. We were also able to specifically interrogate the pathway between the built environment, behaviour (walking) and subsequent mortality.

Our study bears several limitations. The CCHS is a repeated cross-sectional survey and we were unable to track residential moves over the study period. To partially mitigate this limitation, we excluded recent immigrants and imposed a 1-year exposure lag time before ascertaining premature cardiometabolic death. Our measure of ALEs was assessed at one time point. Built environments, especially street networks in North America, are enduring features over the timeframe of decades ${ }^{37}$ and so we believe our reliance on a single-exposure measurement 


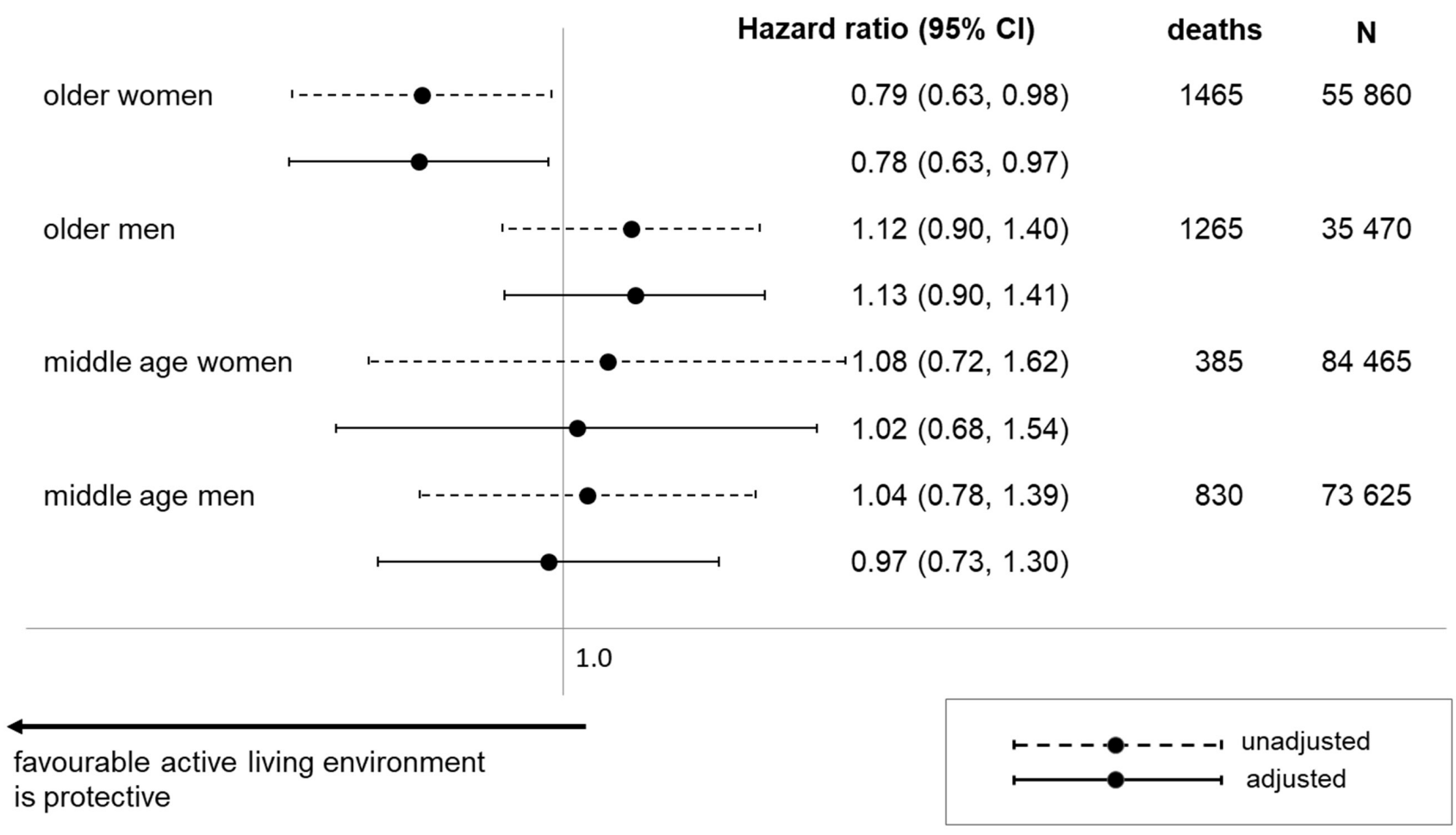

Figure 4 Association of the active living environment with premature cardiometabolic mortality. Data are HRs $(95 \% \mathrm{Cl})$. Unadjusted models (dashed) are adjusted for baseline age. Adjusted models (solid) are adjusted for baseline age, education, income, the presence of two or more chronic conditions, obesity and survey cycle.

time point is justified. While we were also unable to adjust for residential self-selection, ${ }^{38}$ such adjustment has only resulted in minor impacts in some studies examining the associations between the built environment with walking ${ }^{39}$ and obesity. ${ }^{40}$ Our study was limited to self-reported physical activity, which is less precise than objective measures ${ }^{41}$ and is vulnerable to recall bias, especially over longer time frames. ${ }^{42}$ However, such bias is likely to be non-differential with respect to the ALE-an assignment unknown to study participants at survey response. Moreover, the large sample size afforded by multiple waves of a national survey allowed us to overcome the limitations of self-report measures that would likely have generated estimates biased towards the null and helped uncover nationwide physical activity variations that could otherwise be overlooked. Linked environmental, survey and administrative data do not allow us to discern whether reported walking and physical activity take place in one's neighbourhood or not. Previous work using Global Positioning System devices has confirmed that higher levels of physical activity of adults living in more walkable environments are indeed, performed near the home. ${ }^{43}$ As a last point, the reasons why we observe associations in older women and not in other groups could not be easily addressed in our study. We were unable to determine, for instance, whether car use might explain cardiometabolic mortality differences between older women and men. Our large study with linked individual-level data does however serve as a benchmark point for future work to contextualise the association (or lack of association) between the ALE and premature cardiometabolic mortality.

There is growing recognition that the built environment ${ }^{44}$ and urban planning policy ${ }^{45}$ are inherently related to health policy. The ALE is, presumably, a populationlevel exposure, and this study suggests that these environments are likely to encourage population-wide increases in walking and reductions in premature cardiometabolic death. The question as to how we curtail cardiometabolic disease in middle-aged people still stands. Like many places around the world, the Canadian population is ageing, and many are considering ageing in place. Our findings suggest that at present, neighbourhood interventions to support active living are likely to have the greatest gains for older women and can be a viable part of any jurisdiction's approach to preventing premature cardiometabolic death.

Acknowledgements We would like to express our appreciation to Statistics Canada for granting us access to these linked data. We note that the views expressed in this article do not represent those of Statistics Canada.

Contributors SMM, CS, KD and NAR conceptualised the study design. SMM, CS and NAR had full access to the data in the study. SMM performed the statistical analysis. SMM drafted the manuscript. All authors (SMM, CS, KD, MR and NAR) contributed to the data interpretation and revised drafts of the manuscript for important intellectual content. SMM and NAR are the guarantors.

Funding This study was funded by the Canadian Institutes of Health ResearchInstitute of Population and Public Health, through the Data Analysis Existing 
Databases Operating Grant, grant \#410 289. SMM was supported by the Fonds de Recherche du Québec Santé Doctoral Training Award (grant number not applicable). NAR is funded by the Canadian Institutes of Health Research Canada Research Chairs Program.

Disclaimer Funding organisations had no role in the study design, data analysis, interpretation of data, writing of the manuscript and the decision to submit the article for publication.

Competing interests None declared.

Patient consent for publication Not required.

Ethics approval The Canadian Community Health Survey was conducted by Statistics Canada. Ethics approval, and consent to participate in the survey was obtained by Statistics Canada. All analyses were conducted under project number 16-HAD-MCG-4802 at the McGill University site of the Canadian Research Data Centre Network, a secure laboratory which provides access to micro-data holdings of Statistics Canada and has in place a detailed protocol to protect the confidentiality of respondents. Consistent with this protocol, all frequencies have a rounding base to the nearest five respondents, and tabulations resulting in cellcounts under 50 individuals were not released.

Provenance and peer review Not commissioned; externally peer reviewed.

Data availability statement Data may be obtained from a third party and are not publicly available. Data from the Canadian Community Health Survey are available from Statistics Canada for researchers who meet the criteria for access to confidential data. Details can be found at the Statistics Canada Research Data Centres website. See: http://www.statcan.gc.ca/eng/rdc/process

Supplemental material This content has been supplied by the author(s). It has not been vetted by BMJ Publishing Group Limited (BMJ) and may not have been peer-reviewed. Any opinions or recommendations discussed are solely those of the author(s) and are not endorsed by BMJ. BMJ disclaims all liability and responsibility arising from any reliance placed on the content. Where the content includes any translated material, BMJ does not warrant the accuracy and reliability of the translations (including but not limited to local regulations, clinical guidelines, terminology, drug names and drug dosages), and is not responsible for any error and/or omissions arising from translation and adaptation or otherwise.

Open access This is an open access article distributed in accordance with the Creative Commons Attribution Non Commercial (CC BY-NC 4.0) license, which permits others to distribute, remix, adapt, build upon this work non-commercially, and license their derivative works on different terms, provided the original work is properly cited, appropriate credit is given, any changes made indicated, and the use is non-commercial. See: http://creativecommons.org/licenses/by-nc/4.0/.

\section{ORCID iD}

Sarah M Mah http://orcid.org/0000-0002-1533-1203

\section{REFERENCES}

1 Manuel DG, Perez R, Sanmartin C, et al. Measuring burden of unhealthy behaviours using a multivariable predictive approach: life expectancy lost in Canada attributable to smoking, alcohol, physical inactivity, and diet. PLoS Med 2016;13:e1002082.

2 Katzmarzyk PT, Gledhill N, Shephard RJ. The economic burden of physical inactivity in Canada. CMAJ 2000;163:1435-40.

3 Cervero R, Kockelman K. Travel demand and the 3Ds: density, diversity, and design. Transp Res D Transp Environ 1997;2:199-219.

4 Mackenbach JD, Rutter H, Compernolle S, et al. Obesogenic environments: a systematic review of the association between the physical environment and adult weight status, the spotlight project. BMC Public Health 2014;14:233.

5 Chiu M, Rezai M-R, Maclagan LC, et al. Moving to a highly Walkable neighborhood and incidence of hypertension: a Propensity-Score matched cohort study. Environ Health Perspect 2016;124:754-60.

6 Creatore MI, Glazier RH, Moineddin R, et al. Association of neighborhood Walkability with change in overweight, obesity, and diabetes. JAMA 2016;315:2211-20.

7 Sundquist K, Eriksson U, Mezuk B, et al. Neighborhood walkability, deprivation and incidence of type 2 diabetes: a population-based study on 512,061 Swedish adults. Health Place 2015;31:24-30.

8 Patel AV, Hildebrand JS, Leach CR, et al. Walking in relation to mortality in a large prospective cohort of older U.S. adults. Am J Prev Med 2018;54:10-19.

9 Hardy R, Lawlor DA, Kuh D. A life course approach to cardiovascular aging. Future Cardiol 2015;11:101-13.
10 Barrett-Connor E. Gender differences and disparities in all-cause and coronary heart disease mortality: epidemiological aspects. Best Pract Res Clin Endocrinol Metab 2013;27:481-500.

11 YT W, Prina AM, Jones A, et al. Land use mix and five-year mortality in later life: results from the cognitive function and ageing study. Health Place 2016;38:54-60.

12 Hankey S, Marshall JD, Brauer M. Health impacts of the built environment: within-urban variability in physical inactivity, air pollution, and ischemic heart disease mortality. Environ Health Perspect 2012;120:247-53.

13 Griffin BA, Eibner C, Bird CE, et al. The relationship between urban sprawl and coronary heart disease in women. Health Place 2013;20:51-61

14 Gaglioti $\mathrm{AH}, \mathrm{Xu}$ J, Rollins L, et al. Neighborhood environmental health and premature death from cardiovascular disease. Prev Chronic Dis 2018;15:E17.

15 Fecht D, Fortunato L, Morley D, et al. Associations between urban metrics and mortality rates in England. Environ Health 2016;15 Suppl 1:S34.

16 Hamidi S, Ewing R, Tatalovich Z, et al. Associations between urban sprawl and life expectancy in the United States. Int $J$ Environ Res Public Health 2018;15:861.

17 Statistics Canada. Evaluation of the health statistics program, 2015. Available: https://statcan.gc.ca/eng/about/er/hspfr [Accessed 25 Sep 2018].

18 Sanmartin C, Decady Y, Trudeau R, et al. Linking the Canadian community health survey and the Canadian mortality database: an enhanced data source for the study of mortality. Health Rep 2016;27:10-18

19 Canadian Institute for Health Information. Canadian coding standards for CD-10-CA and CCI for 2012. Ottawa: CIHI, 2012

20 World Health Organization. World health statistics 2018: monitoring health for the SDGs sustainable development goals. Geneva: World Health Organization, 2018.

21 Hermann T, Gleckner W, Wasfi RA, et al. A pan-Canadian measure of active living environments using open data. Health Rep 2019;30:16-25

22 Statistics Canada. Canadian community health survey (CCHS) annual component, 2009-2010 common content: derived variable (dv) specifications. Ottawa: Statistics Canada, 2011.

23 Buajitti E, Watson T, Kornas K, et al. Ontario atlas of adult mortality, 1992-2015: trends in local health integration networks, 2018.

24 Connor Gorber S, Shields M, Tremblay MS, et al. The feasibility of establishing correction factors to adjust self-reported estimates of obesity. Health Rep 2008;19:71-82.

25 World Health Organization. Global recommendations on physical activity for health. World Health Organization, 2010.

26 Hajna S, Ross NA, Joseph L, et al. Neighbourhood walkability, daily steps and utilitarian walking in Canadian adults. BMJ Open 2015;5:e008964.

27 Wasfi RA, Dasgupta K, Eluru N, et al. Exposure to walkable neighbourhoods in urban areas increases utilitarian walking: longitudinal study of Canadians. J Transp Health 2016;3:440-7.

28 Morris JN, Hardman AE. Walking to health. Sports Med 1997;23:306-32

29 Hirsch JA, Winters M, Clarke P, et al. Generating GPs activity spaces that shed light upon the mobility habits of older adults: a descriptive analysis. Int J Health Geogr 2014;13:51.

30 Pollard TM, Wagnild JM. Gender differences in walking (for leisure, transport and in total) across adult life: a systematic review. BMC Public Health 2017;17:341.

31 Turcotte M. Profile of seniors' transportation habits. Canadian Social Trends 2012;93:1-16.

32 Althoff T, Sosič R, Hicks JL, et al. Large-Scale physical activity data reveal worldwide activity inequality. Nature 2017;547:336-9.

33 Moore SC, Patel AV, Matthews CE, et al. Leisure time physical activity of moderate to vigorous intensity and mortality: a large pooled cohort analysis. PLoS Med 2012;9:e1001335.

34 Bélanger M, Townsend N, Foster C. Age-Related differences in physical activity profiles of English adults. Prev Med 2011;52:247-9.

35 Pilote L, Dasgupta K, Guru V, et al. A comprehensive view of sex-specific issues related to cardiovascular disease. CMAJ 2007;176:S1-44.

36 Chipperfield JG. Everyday physical activity as a predictor of late-life mortality. Gerontologist 2008;48:349-57.

37 Barrington-Leigh C, Millard-Ball A. A century of sprawl in the United States. Proc Natl Acad Sci U S A 2015;112:8244-9.

38 Boone-Heinonen J, Gordon-Larsen P, Guilkey DK, et al. Environment and physical activity dynamics: the role of residential self-selection. Psychol Sport Exerc 2011;12:54-60. 
39 Handy S, Cao X, Mokhtarian PL. Self-Selection in the relationship between the built environment and walking: empirical evidence from Northern California. J Am Plann Assoc 2006;72:55-74.

40 Sallis JF, Saelens BE, Frank LD, et al. Neighborhood built environment and income: examining multiple health outcomes. Soc Sci Med 2009;68:1285-93.

41 Prince SA, Adamo KB, Hamel ME, et al. A comparison of direct versus self-report measures for assessing physical activity in adults: a systematic review. Int J Behav Nutr Phys Act 2008;5:56.

42 Haskell WL. Physical activity by self-report: a brief history and future issues. J Phys Act Health 2012;9 Suppl 1:S5-10.
43 Hajna S, Kestens Y, Daskalopoulou SS, et al. Neighbourhood walkability and home neighbourhood-based physical activity: an observational study of adults with type 2 diabetes. BMC Public Health 2016;16:957.

44 Tam T. The Chief Public Health Officer's Report on the State of Public Health in Canada 2017-Designing Healthy Living, 2017. Available: https://www.canada.ca/en/public-health/services/publications/ chief-public-health-officer-reports-state-public-health-canada/2017designing-healthy-living.html

45 Giles-Corti B, Vernez-Moudon A, Reis R, et al. City planning and population health: a global challenge. Lancet 2016;388:2912-24. 